\title{
A REMARK ON NELSON'S BEST HYPERCONTRACTIVE ESTIMATES
}

\author{
BARRY SIMON ${ }^{1}$
}

ABSTRACT. By using a combinatorial estimate we provide a new proof of Nelson's best hypercontractive estimates from $L^{2}$ to $L^{4}$.

Let $G$ be the differential operator

$$
-\frac{1}{2} \frac{d}{d x^{2}}+x \frac{d}{d x} \quad \text { on } \quad L^{2}\left(\mathbf{R}, \pi^{-1 / 2} e^{-x^{2}} d x\right) .
$$

Hypercontractive estimates on $e^{-t G}$ have played a key role in constructive quantum field theory; see e.g. [6]. In [5] Nelson proved the estimate

$$
\left\|e^{-t G} f\right\|_{p} \leqslant\|f\|_{q}
$$

if

$$
e^{-t} \leqslant \sqrt{(q-1) /(p-1)}
$$

where $\|\cdot\|_{p}$ is the $L^{p}\left(\mathbf{R}, \pi^{-1 / 2} e^{-x^{2}} d x\right)$ norm. (1) is a "best possible" estimate in the sense that if (2) fails then $e^{-t G}$ is not even bounded from $L^{p}$ to $L^{q}$. Nelson's proof is quite complicated and the beautiful alternate proof of Gross [2] involves some computation. Our goal in this note is to give a simple proof of (1) in case $q=2 ; p=$ even integer. This is not the first time that hypercontractive estimates have been sharper or easier for this case; see the situation for fermions [3].

Our proof proceeds by a slight strengthening of an argument of Nelson [5] who easily proves (1) with $p=4 ; q=2$ if $e^{-t} \leqslant \sqrt{1 / 4}$. Nelson's argument extends to $p=2 k, q=2$ if $e^{-t} \leqslant \sqrt{1 / 2 k}(k=$ integer $)$. Let $A_{k}(n)$ be defined as follows. Consider $2 k n$ objects broken into $2 k$ groups of $n$ objects each. $A_{k}(n)$ is the number of ways of assigning these $2 k n$ objects into $k n$ pairs in such a way that no two objects in the same group are paired with each other. Thus e.g.

$$
A_{1}(n)=n !
$$

Obviously, $A_{k}(n)$ is dominated by the total number of pairings without any restriction and this is $(2 k n) ! /(k n) ! 2^{k n}$. From this one finds that

$$
A_{k}(n) \leqslant(2 k)^{k n}\left(A_{1}(n)\right)^{k} \text {. }
$$

Received by the editors January 17, 1975 and, in revised form, February 25, 1975. AMS (MOS) subject classifications (1970). Primary 81A18, 47D05; Secondary 05A05. Key words and phrases. Hypercontractive.

1 A. Sloan Foundation Fellow; research partially supported by NSF Grant GP 39048. 
(4) is the basis of the easy Nelson proof mentioned above. By mimicking Nelson's proof, the best estimates from $L^{2}$ to $L^{2 k}$ follow from the following combinatorial result which is the main result of this note:

THEOREM 1. $A_{k}(n) \leqslant(2 k-1)^{k n} A_{1}(n)^{k}$.

Proof. We will show that

$$
A_{k n} \leqslant[(2 k-1) / 2 k]^{k n}[(2 k n)(2 k n-2)(2 k n-4) \cdots 2] \text {. }
$$

The last factor in (5) is $2^{k n}(k n)$ !. By the multinomial theorem $(k n)$ ! $\leqslant k^{k n}(n !)^{k}$ so (5) implies the estimate of the theorem. Let us give an algorithm for finding all allowed pairings and then estimate the number of choices at each stage. Write $2 k n$ objects as $\alpha_{1}^{(1)}, \ldots, \alpha_{n}^{(1)}$; $\alpha_{1}^{(2)} \ldots ; \cdots, \alpha_{n}^{(2 k)}$. At each stage choose the group with the most unpaired elements left (if several groups have equal numbers left choose the one with smallest group number $j$ (in $\left.\alpha_{i}^{(j)}\right)$ ). In the group $\alpha^{(j)}$ chosen, pair the $\alpha_{i}^{(j)}$ with $i$ smallest with some element in some other group. This algorithm will clearly yield each allowed pairing once. After $m$ pairs have been chosen, $2 k n-2 m$ elements remain. At least $(2 k n-2 m) / 2 k$ of those elements lie in the group with the most unpaired elements so at the $(m+1) s t$ pairing, at most $[(2 k-1) / 2 k](2 k n-2 m)$ choices are available. This proves the bound (5).

We would also like to make a remark about the best possible nature of the hypercontractive bounds. For a semigroup $e^{-t G}$ taking 1 into 1 , there is a close connection between $G$ having a gap in its spectrum above zero and $e^{-t G}$ being a contraction from $L^{2}$ to $L^{4}$ for some $t$. Glimm [1] proved that if $G$ has a gap and if $e^{-t G}$ is bounded from $L^{2}$ to $L^{4}$ for some $t_{0}$, it is a contraction for sufficiently large $t$. Guerra, Rosen and Simon [4] proved that if $e^{-t G}$ generates a Markov process, then $e^{-t G}$ a contraction from $L^{2}$ to $L^{4}$ implies a mass gap for G. By "running Glimm's proof backwards", we can sharpen the GRS result:

THEOREM 2. Let $T$ be a reality preserving bounded operator on $L^{2}(M ; d \mu)$; $\mu(M)=1$ so that (a) $T 1=1$, (b) $T$ is a contraction from $L^{2}$ to $L^{4}$. Then, $T^{*} 1=1$ and $\left\|T \uparrow\{1\}^{\perp}\right\| \leqslant \sqrt{1 / 3}$.

Proof. Let $f=\alpha 1+g$ with $g \in\{1\}^{\perp}, \alpha$ real and $g$ real valued. Then

$$
\|f\|_{2}^{4}=\left(\alpha^{4}+2 \alpha^{2}\|g\|_{2}^{2}+\|g\|_{2}^{4}\right)
$$

and

$$
\|T f\|_{4}^{4}=\alpha^{4}+4 \alpha^{3}\langle 1, T g\rangle+6 \alpha^{2}\|T g\|_{2}^{2}+O(\alpha) .
$$

By hypothesis: $\|T f\|_{4} \leqslant\|f\|_{2}$ so taking $\alpha$ large we have $\langle 1, T g\rangle \leqslant 0$. This implies that $\langle 1, T g\rangle=0$ so that $T^{*} 1=\alpha 1$. Since $\left\langle 1, T^{*} 1\right\rangle=\langle T 1,1\rangle=1$, $\alpha=1$. Since $\langle 1, T g\rangle=0$, taking $\alpha$ large we have

$$
6\|T g\|_{2}^{2} \leqslant 2\|g\|_{2}^{2} \quad \text { or } \quad\|T g\|_{2} \leqslant \sqrt{1 / 3}\|g\|_{2}
$$

Remark. Thus, the best possible estimate from $L^{2}$ to $L^{4}$ implies that $G$ has a gap of size 1 . If a better estimate held, the gap would be bigger than 1 . Since 
$G$ has a gap of precisely one, we have the best possible nature of the estimates.

It is a pleasure to thank C. Fefferman for a useful remark.

Note. After the completion of this manuscript I learned of two new proofs of the full best hypercontractive estimates, one by W. Beckner and the other by H. Brascamp and E. Lieb.

\section{REFERENCES}

1. J. Glimm, Bosen fields with non-linear self-interaction in two dimensions, Comm. Math. Phys. 8(1968), 12-25.

2. L. Gross, Logarithmic Sobolev inequalities, Amer. J. Math. (to appear).

3. Existence and uniqueness of physical ground states, J. Functional Analysis 10(1972), $52-109$.

4. F. Guerra, L. Rosen and B. Simon, The $P(\phi)_{2}$ quantum field theory as classical statistical mechanics, Ann. of Math. 101 (1975), 111-259.

5. E. Nelson, The free Markoff field, J. Functional Analysis 12(1973), 211-227.

6. B. Simon, The $P(\phi)_{2}$ Euclidean (quantum) field theory, Princeton Univ. Press, Princeton, N.J., 1974.

Departments of Mathematics and Physics, Princeton University, Princeton, New JERSEY 08540 